\title{
PrimeNet - A concept to apply Arrayed Waveguide Grating Multiplexers in a WDM-based fiber backbone
}

\author{
Hagen Woesner \\ Technical University Berlin \\ Telecommunication networks group \\ Einsteinufer 25 \\ 10587 Berlin, Germany \\ Tel.:++49/30/31423825 \\ email:woesner@ee.tu-berlin.de
}

\begin{abstract}
This paper presents a concept for a Multi-Gbit/s WDM-based fiber backbone. The backbone interconnects stations by the means of an Arrayed Waveguide Grating Multiplexer (AWGM). The architecture offers the possibility to evolve from unidirectional virtual ring structures to a fully meshed interconnection. A SONET/SDH based system architecture and a strategy to map ATM service classes on it is proposed.
\end{abstract}

\section{INTRODUCTION}

The basic goal in broadband networking is to deliver high bandwidth at low latency [1]. Optical data transmission is the natural candidate to reach that goal. Over the last few years Wave Division Multiplexing (WDM) has been seen as a proper workaround for the electro-optical bottleneck, i.e. the low electronic processing speed (a few Gbit/s) compared to the high possible bandwidth of a single fiber (several Tbit/s). WDM networks are often recognized as circuit switched networks, because of the long laser tuning times and the aim to provide a "dark fiber" to the end user. The disadvantages of circuit switching are well known (reservation for the peak rate...), but currently outweighed by the plenty of bandwidth WDM is able to provide. This paper emphasizes the combination of Time Division Multiplexing (TDM) and WDM to allow for a packet switched optical network. We develop a concept to interconnect a set of interconnecting devices across an AWGM. The basic idea is to leave the routing decisions within the electronic domain, so that there is no wavelength conversion necessary. While the routing has to be performed in an attached 
(electronic) packet router or switch, the wavelength channel is divided into time slots which can be used through an access protocol. That way a flexible allocation of the bandwidth is made possible in contrast to the use of a circuit based SDH scheme. The basic element of the network is introduced in chapter 2. It enables to set up a logical ring on each wavelength which can be operated independently. The resulting station design and several possibilities to share the bandwidth between the attached stations are shown in chapter 3 and 4, respectively. The crucial issue of connecting several of these networks of several parallel rings is addressed in chapter 5 . We conclude with an outlook on open questions such as the access control protocol and strategies to map different ATM service classes onto paths of different length.

\section{ARRAYED WAVEGUIDE GRATING MULTIPLEXER}

Traditional WDM networks often use passive star couplers to establish single hop networks. While the advantage of single hop networks over multihop approaches in terms of simplicity and transmission delay is obvious, access control protocols are needed to share the bandwidth of the wavelengths. In most cases a separate signalling channel is used, which turns out to be the bottleneck as the number of wavelengths increases. We propose to use a Arrayed Waveguide Grating Multiplexer instead to interconnect the stations of the network. The device and the possible ring structures on it have been described in [2]. The device can be logically seen as a combination of $\mathrm{n}$ demultiplexers and $\mathrm{n}$ multiplexers as in figure 1, though it is essentially an analog grating based element with severe limitations in its size due to crosstalk properties. A wavelength on an input of the AWGM appears only on one output. The advantage of such a solution is that this passive device offers $n$ times the bandwidth of a passive star coupler and it is completely collision free. In principle all routing is done by the selection of the input port and the input wavelength. A signal on wavelength $\lambda 1$ from input $A$ in figure 1 is routed to output $\mathbf{B}^{\mathbf{c}}$, while the same wavelength from input $B$ is routed to output $C^{\prime}$ and from input $C$ to output $\mathrm{A}^{6}$. A station is connected to the AWGM to input $\mathrm{X}$ and to output $\mathrm{X}^{\prime}$, which both have the same relative distance to the in/output of another station. The output port for a certain signal depends on the distance between the receiver and sender and on the selected wavelength. In other words, to select the channel to a specified station, we have to know the distance of the input ports at the AWGM. We lose one wavelength that is routed back to the originating station, so we have (n-1) usable frequencies, each able to reach exactly one of the (n-1) other stations. The Grating Multiplexer has the nice property of being periodic, that is $\lambda \mathrm{x}, \lambda \mathrm{n}+\mathrm{x}, \lambda 2 \mathrm{n}+\mathrm{x} . .$. are all being routed to the same output. The number of periods is limited by the higher attenuation of frequencies far away from the center frequency. This feature allows for the use of more than $n$ wavelengths and may actually enable the parallel transmission of more than one bit. 


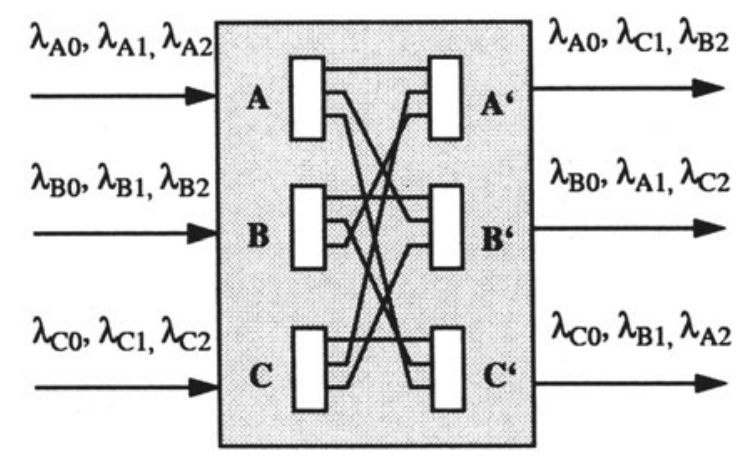

Figure 1 The logical structure of a 3x3 Arrayed Waveguide Grating Multiplexer

\section{BASIC NETWORK STRUCTURE}

In our architecture we use the AWGM in a physical star topology. The proposed basic network structure is a set of virtual rings on the underlaying physical star topology. In figure 2 a network of 3 stations A, B and C is shown. The two wavelengths form a bidirectional ring structure. This can be seen as a fully meshed configuration, too. $\mathrm{A}$ transmission from station $\mathrm{A}$ to $\mathrm{B}$ may take place not only on the "direct" wavelength $\lambda 1$ but also on $\lambda 2$, when a multihop scheme is introduced and station $\mathrm{C}$ works as a relay station for $\mathrm{A}$ and $\mathrm{B}$. We see that potentially all of the wavelengths can be used for a transmission between a given pair of stations. Therefore the overall user data rate for an AWGM with $\mathrm{n}$ inputs (that is, $\mathrm{n}$ stations in maximum) is ( $\mathrm{n}-1$ ) times the bandwidth of a single channel.

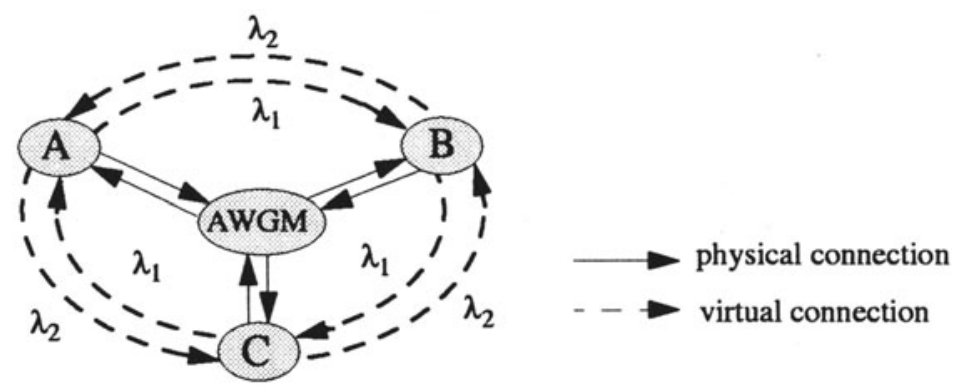

Figure 2 Basic topology of a network made up by a 3x3 AWGM

The routing decision (output wavelength $\lambda$ ) at the sending station is based on:

$\lambda=(x * n+$ distance $) / m=x * n / m+$ distance $/ m$ 
for integer numbers $m, n$ and $x$ with:

$\mathrm{m}=$ hop number $(1<=m<n)$

$\mathrm{n}=$ number of wavelengths

$\mathbf{x}=0 . . \mathrm{n}$

distance $=$ distance between receiver and sender at the input

In a case where $m=x * n$, i.e. $\mathrm{n}$ is an integer multiple of $\mathrm{m}$, there are wavelengths, which can not be used for transmission to a certain station. This is shown in figure 3 , where $\lambda 2$ makes up two separate rings (A-C and B-D) which are not connected to each other, i.e. they do not share a common station. This feature could be used to set up subnetworks, but in our approach we consider it an unwanted effect. Therefore we conclude that the number of wavelengths $n$ in the network and hence the number of inputs of the AWGM has to be a prime number. With $\mathrm{n}$ being a prime number the network consists of (n-1) parallel rings with all stations connected to all rings. We do not consider the periodic nature of the AWGM in the above equation. To take this into account, $x$ would have to go up to $2 \mathrm{n}, 3 \mathrm{n}$ or higher, depending on the number of periods.

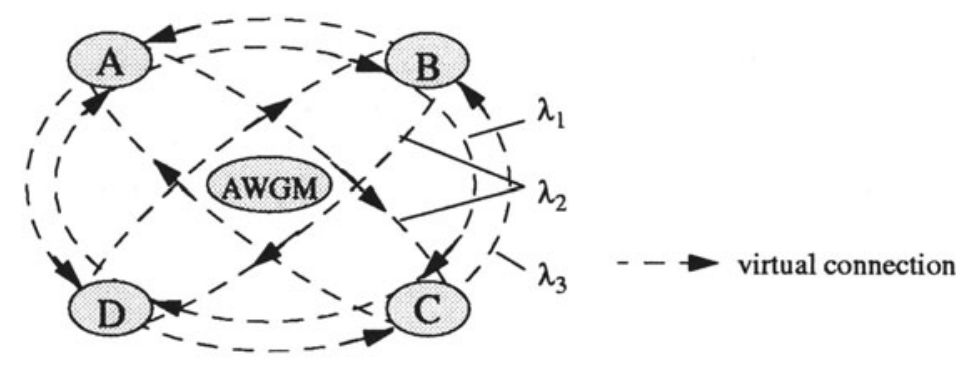

Figure 3 Basic topology of a network made up by a 4x4 AWGM. For better visibility only virtual connections are shown.

\section{DESIGN OF THE NETWORK}

In general, we do not need to have as many Transmitter/Receiver-pairs as wavelengths in the system. It is possible to start up with only one fixed $\mathrm{Tx} / \mathrm{Rx}$ pair per station, which results in a unidirectional ring (e.g. only using $\lambda 1$ in figure 3). By adding additional $\mathrm{Tx} / \mathrm{Rx}$ pairs we increase the possible throughput of each station. We can thereby scale the available bandwidth between two endpoints to the actual needs. If $n$ is a prime number, the wavelengths $\lambda x$ and $\lambda \mathrm{n}-\mathrm{x}(\mathrm{x}=1$...(n-1)/2) form counterdirectional rings. If we assume that we send to one out of (n-1) stations in an unidirectional ring structure, each selected with equal probability, the mean transmission delay relates to $(n-1) / 2$. If we now add a second wavelength to form a bidirectional ring, the delay relates 
to (n-1)/4. So with an increasing number of wavelengths from 1 to (n-1) we reduce the average hop number from $(n-1) / 2$ down to 1 , which is the single hop network.

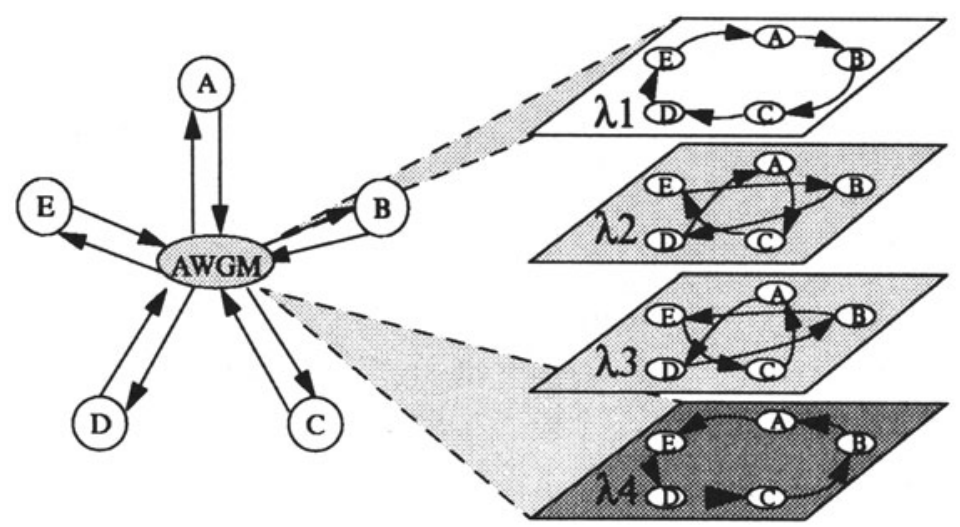

Figure 4 Connections in a network of 5 stations using 4 wavelengths.

There are 3 possible application scenarios for this network: The first possibility is to split the rings up into single point-to-point connections. The second scenario uses SONET/SDH Add-Drop-multiplexers (ADM). A fixed share of the bandwidth is allocated to each connection between two stations. The third and the most flexible approach offers dynamic bandwidth allocation based on a MAC protocol.

\subsection{Point-to-Point Connections}

The simplest possibility would be to put ATM switches into what is referred to as stations in chapter 3 . There would have to be a separate Receiver/Transmitterpair for each input and output, respectively, each on a separate wavelength. So if we would use only one wavelength, the architecture would essentially look like the distributed ATM switch proposed in [4]. On the other hand, if the network is packet switched, the queueing delay for each packet sums up at each switch between the source and destination of the packet. The routing decision could easily be done by evaluating Equation 1. Incoming packets would be routed to the wavelength with the smallest hop number to the destination. If the shortest path is temporarily unavailable the wavelength with the next higher hop number is selected for transmission. A distinction in the routing decision can be made for different ATM service classes. CBR and VBR traffic should always be routed to the shortest path, whereas ABR traffic might take a longer way to its destination.

The strategy of splitting the rings into point-to-point connections is an easily 
applicable way to make use of AWGMs. Still, the bandwidth allocation is not very flexible. For a small number of Transceivers per station the transmission delay of a packet between two stations using the multihop approach could become intolerable.

\subsection{Static Bandwidth Allocation}

The second approach applies Add-Drop-Multiplexers for each wavelength, as the basic functionality of an Add-Drop-Multiplexer is to be a $2 \times 2$ switch. Interestingly, the ideal device to perform demultiplexing and multiplexing on one chip is again the AWGM. A one-chip solution with loop-back optical paths for this is presented in [2]. So the station would employ an AWGM as interface to the optical domain. This increases failure robustness because of its passive nature. It would be possible to have parallel SONET rings with a fixed bandwidth allocation between the stations in a PrimeNet. The station design is shown in figure 5 , where the switch on the left can be of any nature. The ADMs are used to either consume the bandwidth or donate it in short cut mode to the other stations.

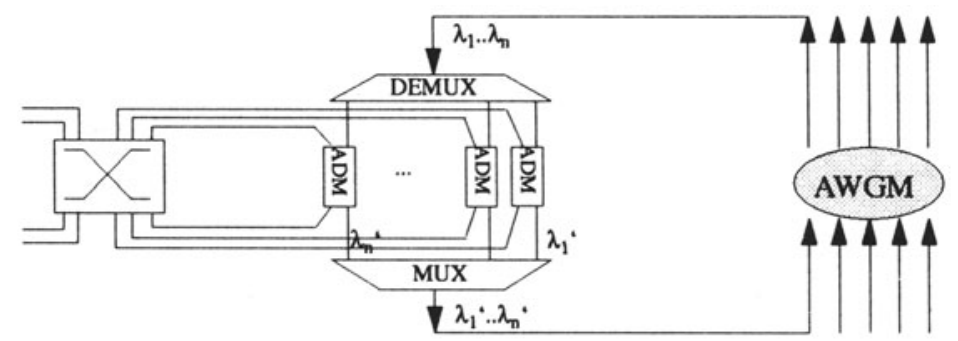

Figure 5 Station design using SONET/SDH rings, $\lambda x$ denotes the input, $\lambda x^{6}$ the output.

\subsection{Dynamic Bandwidth Allocation}

In order to support the bursty nature of packet switched network traffic bandwidth has to be assigned to stations upon their demand. To achieve this we apply a MAC protocol in between the ADM-stage and the switch or router. Here we may apply well-defined solutions like FDDI, DQDB or CRMA [5], that address the problem of fair bandwidth sharing across a ring or a folded bus. The question which of the existing protocols will be appropriate for the needs of the network remains for further studies. The basic requirements for a Media Access Control Protocol in this architecture are: 
- Ability to work equally well for single hop and multihop connections (i.e. independent of the number of rings)

- Low or no reservation overhead in the case of point-to-point connections

- Ability to support QoS - Ability to guarantee bandwidth

The result of the existence of a separate MAC protocol for the network is a new station architecture. It consists of a set of ADMs and a protocol machine to perform routing decisions as well as the actual parallel MAC. A graphical representation for this architecture is shown in figure 6

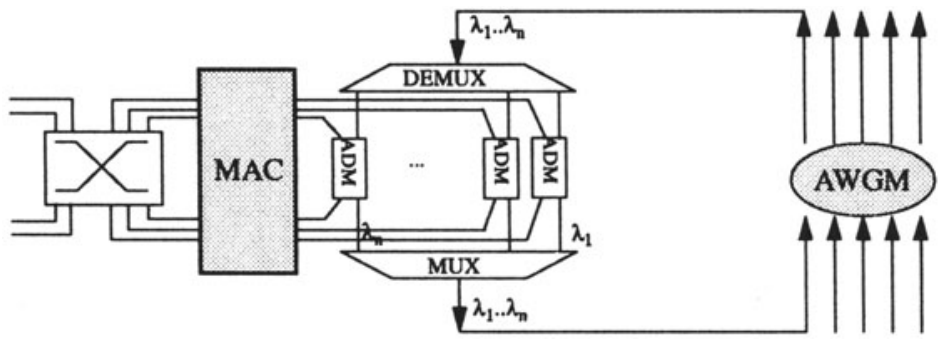

Figure 6 Station design using a parallel MAC machine

\section{EXTENDING THE NETWORK/CASCADING AWGMS}

So far, we only considered a network with a number of stations equal to the number of inputs of the AWGM. These devices are currently on the market up to a size of $64 \times 64$. In order to build networks of, say, 1000 stations or more, we see the need to cascade several AWGMs. A direct connection of AWGMs does not seem to be the proper way because of their modest crosstalk properties. Even if one could employ some kind of signal recovery between the AWGMs, it would physically be the enlargement of every ring on each wavelength involved. So for two networks of 6 stations each (actually 7 stations per network, but one input/output pair is used for the connection to the other AWGM) the direct interconnection of the AWGMs would result in a network of 12 stations on 6 parallel rings, which clearly reduces the available bandwidth for each station. The easiest way to connect two of these networks would be to stick to the point-to-point connections and to have a switch (ATM or IP) which is connected to both. A regular topology which connects every station to two PrimeNets is shown in figure 7. The 16 heptagons represent the PrimeNets that connect the stations. That way we create a robust and redundant topology that eliminates the single point of failure that the AWGM would represent otherwise.

Using this architecture, however, would ask for buffering of data units in the stations. In the case of fixed SONET/SDH bandwidth allocation (chapter 


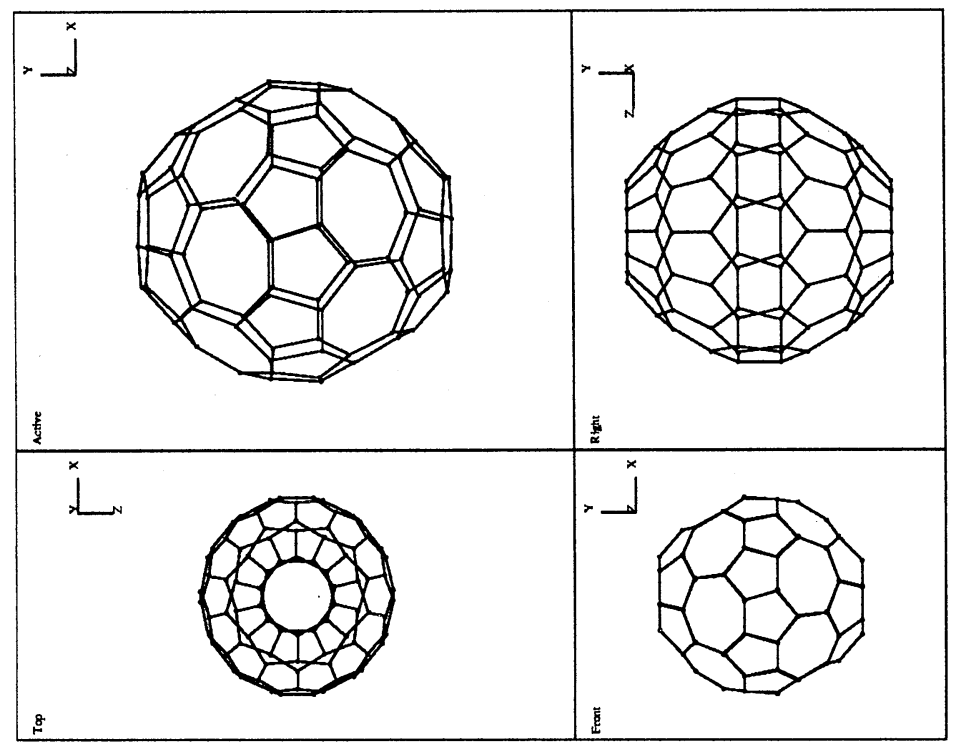

Figure 7 Regular network topology consisting of 16 PrimeNets a 7 switches each

4.2) we would need to have a buffer of at least a SONET/SDH frame size per wavelength to deal with synchronization problems between the two rings. When using the approach of a special parallel MAC protocol, the station connecting the two networks would have to have a second set of ADMs and a second parallel MAC machine. There may be the need for buffering, too, but this depends on the design of the MAC and the reservation policy.

\section{CONCLUSIONS}

We presented a concept for a backbone network interconnecting a number of high-speed switches or routers based on WDM. The WDM concept is translated into a virtual ring structure on every wavelength. That way we can avoid wavelength conversion within the network. By adding $\mathrm{Tx} / \mathrm{Rx}$ pairs in the stations we can scale the available bandwidth from 1 to $(n-1)$ times the bandwidth of a single wavelength, possibly even higher due to the periodic nature of the AWGM (Wavelength $x, n+x, 2 n+x$ are all routed to the same output). Virtual topologies range from an unidirectional ring to a fully meshed topology. The transmission delay decreases with an increasing number of transceivers per station to the optimum case of a point-to-point connection. We discussed several possibilities for the connections on the rings and proposed for a parallel MAC which allows for a flexible bandwidth allocation. The suitability of existing standards like MetaRing, DQDB or CRMA has to be revisited and is a field for further studies. 


\section{REFERENCES}

[1] J.P.G.Sterbenz: Protocols for High Speed Networks: Life After ATM?; Proceedings of IFIP 1995. Protocols for High Speed Networks IV, pp. 3-18; Editors G. Neufeld, M. Ito, Chapman \& Hall, London 1995

[2] Y. Tachikawa, Y. Inoue, M. Ishii, T. Nozawa: Arrayed-Waveguide Grating Multiplexer with Loop-Back Optical Paths and Its Applications; Journal of Lightwave Technology, Vol. 14, No. 6; June 1996

[3] Y. Du, S. Hulyalkar et.al.: System Architecture of a Home Wireless ATM Network, In: Proceedings of ICUPC 1996, San Diego

[4] H.v.As: Media access techniques: The evolution towards terabit/s LANs and MANs; Computer Networks and ISDN Systems 26 (1994); Elsevier Science B.V., 1994 